\title{
Synthesis Antimicrobial and Anticancer Evaluation of 1-Aryl-5-(o-methoxyphenyl)-2-S-benzyl Isothiobiurets
}

\author{
Mohammed M. Ansari, ${ }^{1}$ Shirish P. Deshmukh, ${ }^{1}$ Rizwan Khan, ${ }^{1}$ \\ and Mohammed Musaddiq ${ }^{2}$ \\ ${ }^{1}$ P.G. Department of Chemistry, Shri Shivaji College, Akola, Maharashtra 444001, India \\ ${ }^{2}$ P.G. Department of Microbiology, Shri Shivaji College, Akola, Maharashtra 444001, India \\ Correspondence should be addressed to Mohammed M. Ansari; mohammed.ansari77@gmail.com
}

Received 15 August 2014; Revised 15 October 2014; Accepted 5 November 2014; Published 20 November 2014

Academic Editor: Arie Zask

Copyright (C) 2014 Mohammed M. Ansari et al. This is an open access article distributed under the Creative Commons Attribution License, which permits unrestricted use, distribution, and reproduction in any medium, provided the original work is properly cited.

A series of S-benzyl aryl thiourea were condensed with $o$-Methoxy phenyl isocyanate to yield respective isothiobiuret derivatives. The newly synthesized compounds were characterized by ${ }^{1} \mathrm{H}-\mathrm{NMR}$, IR, and Mass Spectral studies and tested for biological activities.

\section{Introduction}

Thiourea and its derivatives such as thioureides possess interesting biological properties such as antibacterial [1-3], herbicidal, and fungicidal [4]. Many thiourea show in-vivio and in-vitro activity against HIV [5]. An impressive number of currently used drugs can be regarded as thiourea derivatives for example, thyreostatic: carbimazole, propylthiouracil, methylthiouracil, and ultrashortnarcotic: thiamylal. Thiourea shows considerable toxicity towards higher organisms and is used as insecticide [6] and rodenticide [7]. Thiourea derivatives find widespread uses in mining industry as floating aid for sulfidic ores [8].

Thiobiurets (mono and di) are important derivatives of (thio) urea which can increase the biological activity of (thio) ureas. Mono and dithiobiuret derivatives are effective bactericide, fungicide, herbicide, and miticides [9]. Dithiobiuret derivatives are used for repelling birds, rodents, leporine animals, and ruminants [10]. 1-Allyl-2-thiobiuret regulates the growth of germinating wheat and cucumber seeds [11]. Oliver and coworkers $[12,13]$ reported chemosterilising action of dithiobiuret derivatives in male house flies. Mono and dithiobiuret showed effective growth regulating activity [14]. Thiobiuret derivatives also showed analgesic
[15], anticonvulsant, and hypnotic activity [16]. Glycosyl urea and their biuret derivatives are reported as potential glycoenzyme inhibitors [17]. Dandale and Deshmukh [18] reported antibacterial and antifungal activities of per $\mathrm{O}$ acetylated lactosyl monothiobiurets.

In quest for biologically more potent compounds, we envisioned synthesizing series of isothiobiuret compounds by reacting $S$-benzyl arylthiourea with $o$-methoxy phenyl isocyanate and studied their antibacterial and anticancer activities.

\section{Result and Discussion}

\subsection{Chemistry}

2.1.1. Experimental. Melting points were recorded on electrothermal melting point apparatus. IR spectra were recorded on a Shamazdu FTIR spectrometer. ${ }^{1} \mathrm{H}$ NMR was obtained on a Bruker DRX-300 (300 MHz FT NMR) NMR spectrometer in $\mathrm{CDCl}_{3}$ solution with TMS as an internal reference. The mass spectra were recorded on a Jeol SX-102 FAB mass spectrometer. Purity of the compounds was checked by thin layer chromatography using Merck silica gel coated aluminum plates and petroleum ether: ethyl acetate as eluent. 


\subsection{General Scheme of Synthesis}

Step 1. Thiourea synthesis: (see Scheme 1).

Step 2. S-Benzylation: (see Scheme 2).

Step 3. Thiourea isocyanate condensation: (see Scheme 3).

\subsection{Synthetic Procedure}

2.3.1. Synthesis of Aryl S-benzyl Thiourea. General synthetic procedure for preparation of Aryl thiourea exemplified by phenyl thiourea: Aniline $(30 \mathrm{~g}, 0.32 \mathrm{~mol})$ was taken in a round bottom flask and, to this, concentrated hydrochloric acid $(32.19 \mathrm{~mL}, 0.32 \mathrm{~mol})$ was added dropwise with continues stirring. After $20 \mathrm{~min}$ turbidity appeared and $100 \mathrm{~mL}$ of water was added followed by a solution of ammonium thiocyanate $(29.42 \mathrm{~g}, 0.38 \mathrm{~mol})$ in $50 \mathrm{~mL}$ of water. This reaction mixture was heated till the solution starts becoming turbid, heating discontinued, reaction mass was poured in ice cold water, and precipitate formed was filtered off and dried. Crude product was recrystallized by hot water, yield (39.22 g, 80\%), m.p. $152^{\circ} \mathrm{C}$.

General synthetic procedure for preparation of Aryl Sbenzyl thiourea exemplified by phenyl $S$-benzyl thiourea (II): phenyl thiourea $(35 \mathrm{~g}, 0.23 \mathrm{~mol})$ was dissolved in $75 \mathrm{~mL}$ of ethanol and, to this reaction, mass benzyl chloride $(29.11 \mathrm{~mL}$, $0.25 \mathrm{~mol}$ ) was added; once the exotherm subsides, reaction mass was gently heated to reflux and refluxed for $90 \mathrm{~min}$. This reaction mixture was cooled to room temperature and basified under cold condition with ammonia solution, and, on standing, it yields phenyl S-benzyl thiourea. Yield (29.2 g, $52.4 \%)$, m.p. $75^{\circ} \mathrm{C}$.

2-Methoxy phenyl isocyanate was procured from Sigma Aldrich.

General synthetic procedure for preparation of 1-Aryl-5(o-methoxyphenyl)-2-S-benzyl isothiobiuret was exemplified using 1-Phenyl-5-(o-methoxyphenyl)-2-S-benzyl isothiobiuret.

1-Phenyl-5-(o-methoxyphenyl)-2-S-benzyl Isothiobiuret. Isothiobiuret was synthesized by condensing Phenyl S-benzyl thiourea $(0.70 \mathrm{~g}, 2.89 \mathrm{mmole})$ with $o$-Methoxy phenyl isocyanate $(0.43 \mathrm{~g}, 2.89 \mathrm{mmole})$ at room temperature in benzene overnight. Solvent was removed by distillation. Solid mass obtained was triturated with pet ether, to afford off-white solid.

Molecule Number 1: 1-Phenyl-5-(o-methoxyphenyl)-2-S-benzyl Isothiobiuret. Obtain as off white solid (87.12\%) m.p. 100$102^{\circ} \mathrm{C}$, TLC $R_{f} 0.7$ in EtOAc: Petether (3:7) visualized using iodine, IR (KBr) in $\mathrm{cm}^{-1} v 3290(\mathrm{~N}-\mathrm{H}) ; 2906(\mathrm{Ar}-\mathrm{H}) ; 2839$ (Methoxy); $1670(\mathrm{C}=\mathrm{O}) ; 1236(\mathrm{C}-\mathrm{N}) .{ }^{1} \mathrm{H}-\mathrm{NMR}(300 \mathrm{MHz}$, $\left.\mathrm{CDCl}_{3}\right) \delta: 3.9(\mathrm{~s}, 3 \mathrm{H}), 4.4(\mathrm{~s}, 2 \mathrm{H}), 6.9-7.8(\mathrm{~m}, 14 \mathrm{H}), 8.3$ (s, 1H), 11.8 (s, 1H). MS, m/z: $392\left[\mathrm{M}^{+}+1\right]$, Anal. Calcd. for $\mathrm{C}_{22} \mathrm{H}_{21} \mathrm{~N}_{3} \mathrm{O}_{2} \mathrm{~S}$, Requires: C: $67.50 \%$, H: $5.41 \%$, N: $10.73 \%$, S: 8.19\% Found: C: $67.71 \%$, H: 5.21\%, N: $10.00 \%$, S: $8.00 \%$.
Molecule Number 2: 1-(p-Tolyl)-5(o-methoxyphenyl)-2-Sbenzyl Isothiobiuret. Obtain as off white solid (60.06\%) m.p. $105-108^{\circ} \mathrm{C}$, TLC $R_{f} 0.7$ in EtOAc: Petether $(3: 7)$ visualized using iodine, IR (KBr) in $\mathrm{cm}^{-1} v 3300(\mathrm{~N}-\mathrm{H}) ; 2960(\mathrm{Ar}-\mathrm{H})$; 2839 (Methoxy); $1741(\mathrm{C}=\mathrm{O}) .{ }^{1} \mathrm{H}-\mathrm{NMR}\left(300 \mathrm{MHz}, \mathrm{CDCl}_{3}\right.$ ) $\delta: 2.4(\mathrm{~s}, 3 \mathrm{H}), 3.8(\mathrm{~s}, 3 \mathrm{H}), 4.5(\mathrm{~s}, 2 \mathrm{H}), 6.89(\mathrm{~d}, 2 \mathrm{H}), 7.0-7.6$ (m, 11H), 8.1 (s, 1H), 11.5 (s, 1H). MS, m/z: $406\left[\mathrm{M}^{+}+1\right]$, Anal. Calcd. for $\mathrm{C}_{23} \mathrm{H}_{23} \mathrm{~N}_{3} \mathrm{O}_{2}$ S, Requires: C: $68.12 \%, \mathrm{H}: 5.72 \%, \mathrm{~N}$ : 10.36\%, O: 7.89\%, S: 7.91\% Found: C: $67.10 \%, \mathrm{H}: 5.15 \%, \mathrm{~N}$ : $10.00 \%$, S: $8.00 \%$.

Molecule Number 3: 1-(o-Tolyl)-5-(o-methoxyphenyl)-2-Sbenzyl Isothiobiuret. Obtain as off white solid (60.50\%) m.p. 95-97 ${ }^{\circ} \mathrm{C}$, TLC $R_{f} 0.7$ in EtOAc: Petether $(3: 7)$ visualized using iodine, IR $(\mathrm{KBr})$ in $\mathrm{cm}^{-1} v 3250(\mathrm{~N}-\mathrm{H}) ; 2895(\mathrm{Ar}-\mathrm{H})$; $1720(\mathrm{C}=\mathrm{O}) ; 1610(\mathrm{C}=\mathrm{N}) ; 1350(\mathrm{C}-\mathrm{N}) ; 1235(\mathrm{C}-\mathrm{O}) .{ }^{1} \mathrm{H}-\mathrm{NMR}$ $\left(300 \mathrm{MHz} \mathrm{CDCl}_{3}\right) \delta: 2.4(\mathrm{~s}, 3 \mathrm{H}), 3.9(\mathrm{~s}, 3 \mathrm{H}), 4.4(\mathrm{~s}, 2 \mathrm{H}), 6.9$ $(\mathrm{t}, 1 \mathrm{H}), 7.0(\mathrm{t}, 2 \mathrm{H}), 7.1(\mathrm{~d}, 2 \mathrm{H}), 7.2-7.7(\mathrm{~m}, 8 \mathrm{H}), 8.0(\mathrm{~s}, 1 \mathrm{H}), 11.7$ (s, $1 \mathrm{H})$. MS, m/z: $406\left[\mathrm{M}^{+}+1\right]$, Anal. Calcd. for $\mathrm{C}_{23} \mathrm{H}_{23} \mathrm{~N}_{3} \mathrm{O}_{2} \mathrm{~S}$, Requires: C: $68.12 \%$, H: 5.72\%, N: 10.36\%, O: 7.89\%, S: $7.91 \%$. Found: C: $69.30 \%, \mathrm{H}: 5.00 \%, \mathrm{~N}: 10.20 \%$, S: $7.50 \%$.

Molecule Number 4: 1-(p-Cl-phenyl)-5-(o-methoxyphenyl)-2S-benzyl Isothiobiuret. Obtain as off white solid (63.55\%) m.p. $122-124^{\circ} \mathrm{C}$, TLC $R_{f} 0.8$ in EtOAc: Petether $(3: 7)$ visualized using iodine, IR (KBr) in $\mathrm{cm}^{-1} v 3250(\mathrm{~N}-\mathrm{H}) ; 2850(\mathrm{Ar}-\mathrm{H})$; $1700(\mathrm{C}=\mathrm{O}) ; 1600(\mathrm{C}=\mathrm{N}) ; 1370(\mathrm{C}-\mathrm{N}) ; 1235(\mathrm{C}-\mathrm{O}) .{ }^{1} \mathrm{H}-\mathrm{NMR}$ $\left(300 \mathrm{MHz} \mathrm{CDCl}_{3}\right) \delta: 3.8(\mathrm{~s}, 3 \mathrm{H}), 4.5(\mathrm{~s}, 2 \mathrm{H}), 7.0-7.2(\mathrm{~m}, 5 \mathrm{H})$, 7.3-7.4 (m, 5H), 7.5 (m, 3H), $11.4(\mathrm{~s}, 1 \mathrm{H})$. MS, m/z: $427\left[\mathrm{M}^{+}+1\right]$, Anal. Calcd. for $\mathrm{C}_{22} \mathrm{H}_{20} \mathrm{ClN}_{3} \mathrm{O}_{2} \mathrm{~S}$, Requires: C: $62.04 \%, \mathrm{H}$ : 4.73\%, Cl: $8.32 \%$, N: 9.87\%, O: 7.51\%, S: 7.53\%. Found: C: 58.71\%, H: 5.40\%, N: $9.00 \%$, S: 7.00\%, Cl: 7.50\%.

Molecule Number 5: 1-(o-Cl-phenyl)-5-(o-methoxyphenyl)-2S-benzyl Isothiobiuret. Obtain as off white solid (75.14\%) m.p. $118-120^{\circ} \mathrm{C}$, TLC $R_{f} 0.8$ in EtOAc: Petether $(3: 7)$ visualized using iodine, IR (KBr) in $\mathrm{cm}^{-1} v 3300(\mathrm{~N}-\mathrm{H}) ; 2960(\mathrm{Ar}-\mathrm{H})$; $1741(\mathrm{C}=\mathrm{O}) ; 1590(\mathrm{C}=\mathrm{N}) ; 1372(\mathrm{C}-\mathrm{N}) ; 1235(\mathrm{C}-\mathrm{O}) .{ }^{1} \mathrm{H}-\mathrm{NMR}$ $\left(300 \mathrm{MHz} \mathrm{CDCl}_{3}\right) \delta: 3.6(\mathrm{~s}, 3 \mathrm{H}), 4.4(\mathrm{~s}, 2 \mathrm{H}), 7.0-7.1(\mathrm{~m}, 3 \mathrm{H})$, 7.3-7.4 (m, 8H), 7.5-7.6 (d, 2H), 8.1 (s, 1H), 11.3 (s, 1H). MS, $\mathrm{m} / \mathrm{z}: 427\left[\mathrm{M}^{+}+1\right]$, Anal. Calcd. for $\mathrm{C}_{22} \mathrm{H}_{20} \mathrm{ClN}_{3} \mathrm{O}_{2} \mathrm{~S}$, Requires: C: $62.04 \%, \mathrm{H}: 4.73 \%, \mathrm{Cl}: 8.32 \%$, N: 9.87\%, O: 7.51\%, S: 7.53\%. Found: C: $60.40 \%$, H: $4.40 \%, \mathrm{~N}: 9.00 \%$, S: $7.00 \%$, Cl: $7.50 \%$.

Molecule Number 6: 1-(m-Cl-phenyl)-5-(o-methoxyphenyl)-2S-benzyl Isothiobiuret. Obtain as off white solid (77.60\%) m.p. $105-107^{\circ} \mathrm{C}$, TLC $R_{f} 0.8$ in EtOAc: Petether (3:7) visualized using iodine, IR (KBr) in $\mathrm{cm}^{-1} v 3280(\mathrm{~N}-\mathrm{H}) ; 2900(\mathrm{Ar}-$ $\mathrm{H}) ; 1670(\mathrm{C}=\mathrm{O}) ; 1550(\mathrm{C}=\mathrm{N}) ; 1320(\mathrm{C}-\mathrm{N}) ; 1230(\mathrm{C}-\mathrm{O}) .{ }^{1} \mathrm{H}-$ $\mathrm{NMR}\left(300 \mathrm{MHz}, \mathrm{CDCl}_{3}\right) \delta: 3.8(\mathrm{~s}, 3 \mathrm{H}), 4.5(\mathrm{~s}, 2 \mathrm{H}), 6.8-7.1$ (m, 4H), 7.3-7.4 (m, 5H), 7.4-7.5 (m, 2H), 7.8-7.9 (m, 2H), 8.3 (s, $1 \mathrm{H}), 11.5$ (s, 1H). MS, m/z: $427\left[\mathrm{M}^{+}+1\right]$, Anal. Calcd. for $\mathrm{C}_{22} \mathrm{H}_{20} \mathrm{ClN}_{3} \mathrm{O}_{2} \mathrm{~S}$, Requires: C: $62.04 \%, \mathrm{H}: 4.73 \%$, Cl: $8.32 \%$, N: $9.87 \%$, O: $7.51 \%$, S: $7.53 \%$. Found: C: $65.21 \%, \mathrm{H}: 4.40 \%, \mathrm{~N}$ : $8.70 \%$, S: $7.00 \%, \mathrm{Cl}: 7.60 \%$. 


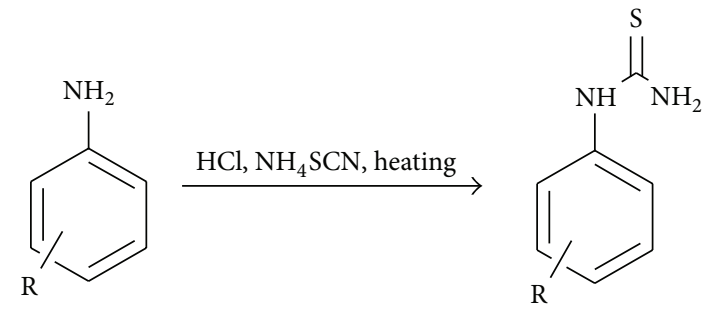

Where, $\mathrm{R}$ is H, 4-Chloro, 2-Chloro, 3-Chloro, 2-Methyl and 4-Methyl.

SCHEMe 1

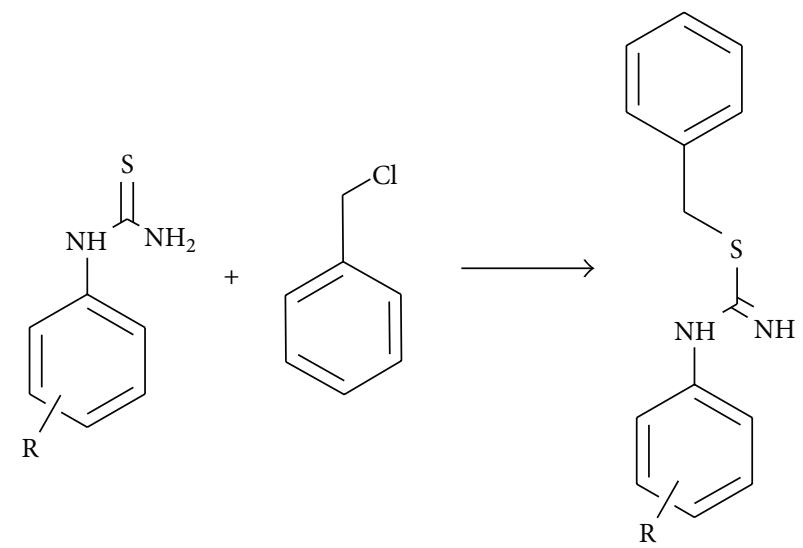

Where, $\mathrm{R}$ is H, 4-Chloro, 2-Chloro, 3-Chloro, 2-Methyl and 4-Methyl.

SCHEME 2<smiles>[R]c1cccc(N=C(NC(=O)Nc2ccccc2OC)SCc2ccccc2)c1</smiles>

Where, $\mathrm{R}$ is H, 4-Chloro, 2-Chloro, 3-Chloro, 2-Methyl and 4-Methyl.

SCHEME 3

2.4. Antimicrobial Activity. All the compounds were screened for their antibacterial activity against pathogenic bacteria and fungi such as E. coli, S. aureus, P. aeruginosa, and Aspergillus fusarium by cup plate agar diffusion method at a concentration $100 \mu \mathrm{g} / \mathrm{mL}$ in DMSO. The zone of inhibition was measured in $\mathrm{mm}$ and is average of three readings. The readings are shown in Table 1.

Molecule 4 showed moderate antimicrobial activity against E. coli and S. aureus, and considerable antifungal activity, whereas molecule number 2 showed a reverse trend in activities; from this observation, it can be concluded that substitution at para position of phenyl ring plays a crucial role in deciding activity toward bacterial and fungal stains.
2.5. Anticancer Activity. Molecule number 1 as representative molecule was studied for short term in vitro cytotoxicity using Dalton's ascites (DLA) cells and Ehrlich ascites Carcinoma (EAC) Cells.

The tumor cells aspirated from the peritoneal cavity of tumor bearing mice were washed thrice with phosphate buffered saline (PBS) or normal saline. Cell viability was determined by trypan blue exclusion method, viable cell suspension $\left(1 \times 10^{6}\right.$ cells in $\left.0.1 \mathrm{~mL}\right)$ was added to tubes containing various concentrations of the test compounds, and the volume was made up to $1 \mathrm{~mL}$ using PBS. Control tube contained only cell suspension; these assay mixtures were incubated for 3 hours at $37^{\circ} \mathrm{C}$. Further cell suspension 
TABLE 1: Antimicrobial activities of molecules numbers 1 to 6 .

\begin{tabular}{lcccc}
\hline Molecule number & E. coli & $\begin{array}{c}\text { Antibacterial activity } \\
\text { S. aureus }\end{array}$ & P. aeruginosa & $\begin{array}{c}\text { Antifungal activity } \\
\text { Aspergillus fusarium }\end{array}$ \\
\hline 1 & 12 & 8 & 15 & 10 \\
2 & 18 & 18 & 19 & 12 \\
3 & 18 & 10 & 10 & 10 \\
4 & 12 & 12 & 8 & 18 \\
5 & 10 & 17 & 16 & 8 \\
6 & 10 & 18 & 23 & NA \\
Amikacin & 25 & 23 & NA & 23 \\
Ketoconazole & NA & NA & Nil & Nil \\
Control (DMSO) & Nil & Nil & & 10 \\
\hline
\end{tabular}

Including well diameter of $5 \mathrm{~mm}$.

TABLE 2: Anticancer activity of molecule number 1.

\begin{tabular}{lccc}
\hline Compound number & Drug concentration $(\mu \mathrm{g} / \mathrm{mL})$ & Percentage cell death (DLA) & Percentage cell death (EAC) \\
\hline & 200 & 64 & 70 \\
& 100 & 40 & 56 \\
Molecule 1 & 50 & 26 & 36 \\
& 20 & 13 & 6 \\
\hline & 10 & 6 & 92 \\
5-Fluorouracil & 100 & NA & NA \\
& 50 & 97 & 29 \\
& 20 & NA & NA \\
\hline
\end{tabular}

5-Fluorouracil was used as a standard. Molecule 1 shows considerable cell toxicity at 50 and $20 \mu \mathrm{g}$ concentration.

was mixed with $0.1 \mathrm{~mL}$ of $1 \%$ trypan blue and kept for $2-3$ minutes and loaded on a haemocytometer. Dead cells take up the blue colour of trypan while live cells do not take up the dye. The numbers of stained and unstained cells were counted separately; drug concentration versus percentage of death cells was tabulated in Table 2:

\section{\%Cytotoxicity}

$$
=\frac{\text { Number of dead cells }}{\text { Number of live cells }+ \text { Number of dead cells }} \times 100 \text {. }
$$

\section{Conclusions}

From the observation, it can be concluded that substitution at para position of phenyl ring plays a crucial role in deciding activity toward bacterial and fungal stain; as these molecules are easy to synthesize and purify, these classes of molecules can be explored further to develop SAR against different microbial and fungal stains as well as a potent anticancer agent.

\section{Conflict of Interests}

The authors declare that there is no conflict of interests regarding the publication of this paper.

\section{Acknowledgments}

The authors thank Sophisticated Analytical Instrumentation Facility (SAIF), a division of Central Drug Research Laboratory (CDRI) Lucknow for recording spectra, Dr. Ramadasan Kuttan Research Director Amala Cancer Research Centre Thrissur Kerala for providing cytotoxicity profiling of molecules and Dr. S G Bhadange Principal Shri Shivaji College of Science Akola for providing necessary facilities.

\section{References}

[1] Z. Kazimierczuk, M. Chalimoniuk, A. E. Laudy et al., "Synthesis and antimicrobial and nitric oxide synthase inhibitory activities of novel isothiourea derivatives," Archives of Pharmacal Research, vol. 33, no. 6, pp. 821-830, 2010.

[2] E. G. Chalina and L. Chakarova, "Synthesis, hypotensive and antiarrhythmic activities of 3-alkyl-1-(2-hydroxy-5,8-dimetho$\mathrm{xy}$-1,2,3,4-tetrahydro-3-naphthalenyl)ureas or thioureas and their guanidine analogues," European Journal of Medicinal Chemistry, vol. 33, no. 12, pp. 975-983, 1998.

[3] H. Stark, K. Purand, X. Ligneau et al., "Novel carbamates as potent histamine $\mathrm{H}_{3}$ receptor antagonists with high in vitro and oral in vivo activity," Journal of Medicinal Chemistry, vol. 39, no. 5, pp. 1157-1163, 1996.

[4] C. Walpole, S. Y. Ko, M. Brown et al., "2-Nitrophenylcarbamoyl(S)-prolyl-(S)-3-(2-naphthyl)alanyl- $N$-benzyl- $N$ - methylamide (SDZ NKT 343), a potent human $\mathrm{NK}_{1}$ tachykinin receptor antagonist with good oral analgesic activity in chronic pain 
models," Journal of Medicinal Chemistry, vol. 41, no. 17, pp. 31593173, 1998.

[5] S. B. Tsogoeva, M. J. Hateley, D. A. Yalalov, K. Meindl, C. Weckbecker, and K. Huthmacher, "Thiourea-based nonnucleoside inhibitors of HIV reverse transcriptase as bifunctional organocatalysts in the asymmetric Strecker synthesis," Bioorganic and Medicinal Chemistry, vol. 13, no. 19, pp. 56805685, 2005.

[6] F. Ruder and H. Kayser, "The thiourea insecticide diafenthiuron inhibits mitochondrial ATPase in vitro and in vivo by its carbodiimide product," Biochemical Society Transactions, vol. 22, no. 1, pp. 241-244, 1994.

[7] J. C. Kawalek, A. W. Andrews, and R. J. Pienta, "1-Naphthylthiourea: a mutagenic rodenticide that transforms hamster embryo cells," Molecular Pharmacology, vol. 15, no. 3, pp. 678684, 1979.

[8] F. Z. El Aamrani, A. Kumar, J. L. Cortina, and A. M. Sastre, "Solvent extraction of copper(II) from chloride media using $\mathrm{N}$ (thiocarbamoyl)benzamidine and $\mathrm{N}$-benzoylthiourea derivatives," Analytica Chimica Acta, vol. 382, no. 1-2, pp. 205-213, 1999.

[9] R. F. Bellina, US 4010199, 1977.

[10] G. Hermann, P. Hoffmann, and F. Rosendahl, "Dithiobiuret derivatives used for repelling birds, rodents, leporine animals and ruminants," I. Ugi. US 3941887A, 1976.

[11] S. N. Pandeya, A. Kumar, B. N. Singh, and D. N. Mishra, "Synthesis and biological activity of isodithiobiurets, dithiobiurets, and dithiazoles," Pharmaceutical Research, vol. 4, no. 4, pp. 321326, 1987.

[12] J. E. Oliver, S. C. Chang, R. T. Braun, and A. B. Borkovic, "Insect chemosterilants. 10. Substituted dithiobiurets," Journal of Medicinal Chemistry, vol. 14, pp. 772-773, 1971.

[13] J. E. Oliver, R. T. Brown, R. L. Fye, and A. B. Borkovec, "Insect chemosterilants. 1,2,4-Dithiazolium salts and related compounds as additives to housefly diet," Journal of Agricultural and Food Chemistry, vol. 21, no. 5, pp. 753-755, 1973.

[14] F. Obrero and W. H. Pschnitzler, US 3741746, 1973.

[15] J. Zielinski and N. J. Kenilworth, "Use of dithiobiurets as fungicides," U.S. Patent no. US3818104 A, 1974.

[16] N. Siddiqui and A. Husain, "Some substituted thiobiurets as possible analgesic agents," Indian Journal of Pharmacology, vol. 33, no. 5, pp. 382-383, 2001.

[17] N. Felföldi, M. Tóth, E. D. Chrysina, M.-D. Charavgi, K.-M. Alexacou, and L. Somsák, "Synthesis of new glycosyl biuret and urea derivatives as potential glycoenzyme inhibitors," Carbohydrate Research, vol. 345, no. 2, pp. 208-213, 2010.

[18] A. S. Dandale and S. P. Deshmukh, "Synthesis and antimicrobial activity of N-lactosylated isothiobiurets," Journal of the Indian Chemical Society, vol. 84, no. 12, pp. 1266-1268, 2007. 

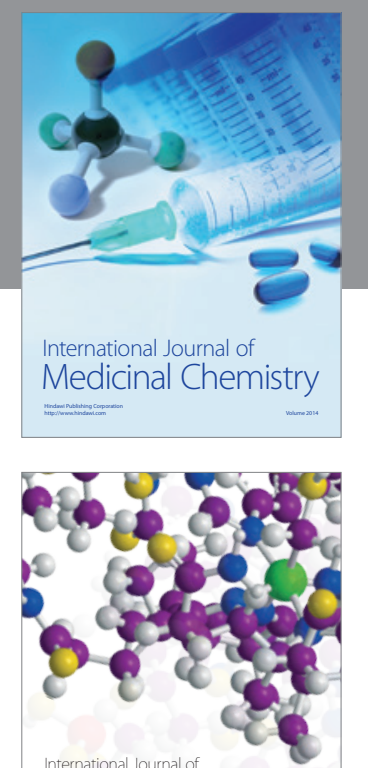

\section{Carbohydrate} Chemistry

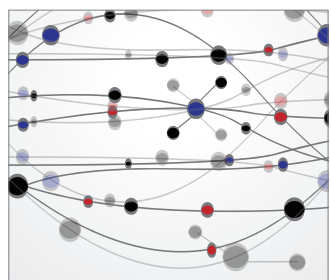

The Scientific World Journal
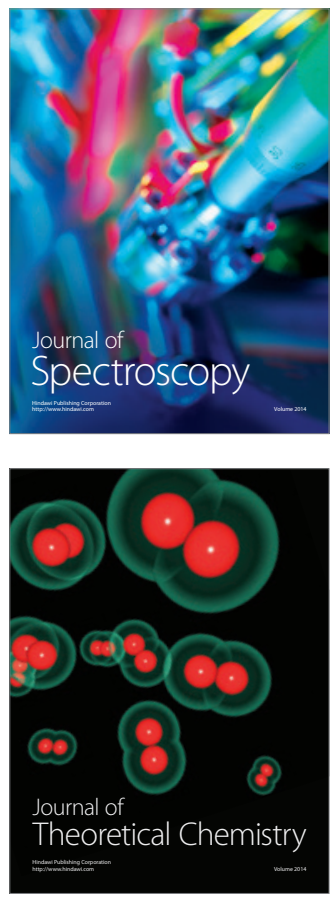
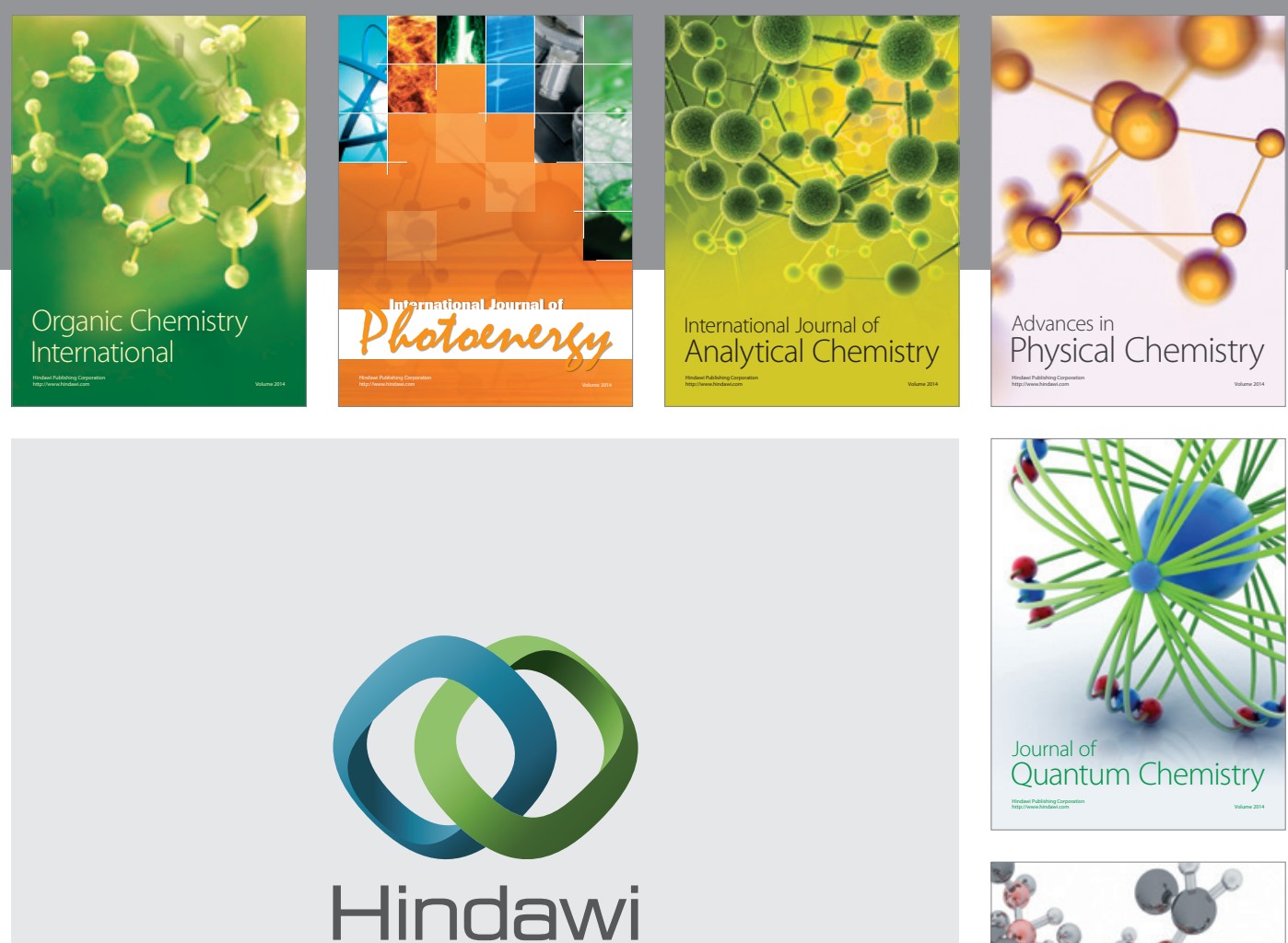

Submit your manuscripts at

http://www.hindawi.com

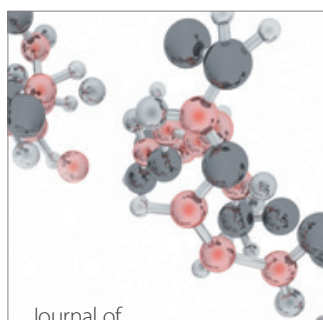

Analytical Methods

in Chemistry

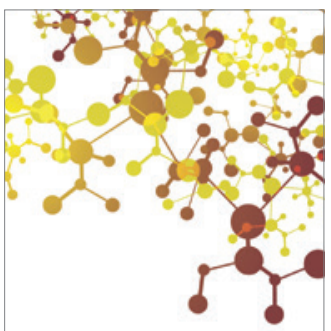

Journal of

Applied Chemistry

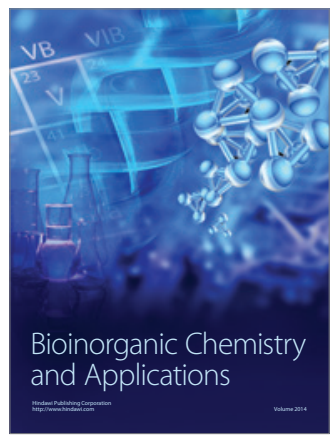

Inorganic Chemistry
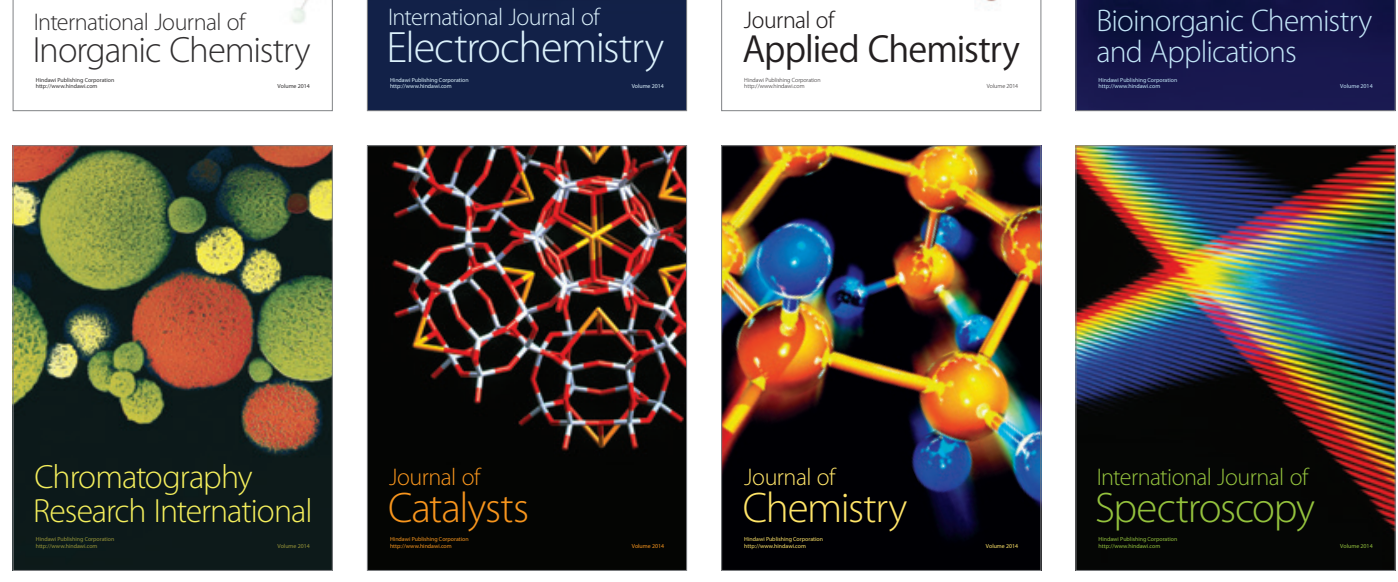